\title{
PROPIEDADES MECÁNICAS DE MEZCLAS BITUMINOSAS EN CALIENTE FABRICADAS CON ÁRIDOS RECICLADOS DE RESIDUOS DE CONSTRUCCIÓN Y DEMOLICIÓN
}

\author{
Pérez $^{+}, \mathrm{I}$; Toledano ${ }^{+}, \mathrm{M}$; Gallego ${ }^{*}, \mathrm{~J} ;$ Taibo $^{+}, \mathrm{J}$ \\ +Universidade da Coruña. E. T. S. I. Caminos, Campus de Elviña, s/n, 15071 A Coruña \\ *Universidad Politécnica de Madrid. E. T. S. I. Caminos, Profesor Aranguren, s/n, 28040 Madrid
}

Fecha de envío: 27 de diciembre 2005

Fecha de segundo envío: 26 de junio 2006

\begin{abstract}
Resumen
En este trabajo se dosificaron mezclas bituminosas fabricadas con áridos reciclados de residuos de construcción y demolición. Se realizaron una serie de ensayos que permitieron caracterizar el comportamiento mecánicos de estas mezclas. Los parámetros mecánicos de las mezcla con áridos reciclados son similares a los de las mezclas fabricadas únicamente con áridos naturales de cantera. Sin embargo, las mezclas bituminosas con áridos reciclados tuvieron un mal comportamiento frente a la disminución de la resistencia por pérdida de cohesión por la acción del agua. No obstante, mediante la selección de materiales de mejor calidad y corrección de este mal comportamiento, existen posibilidades de utilizar áridos reciclados en mezclas bituminosas. Para ello será necesario ampliar la primera aproximación realizada en este artículo.
\end{abstract}

Palabras clave: Residuos de construcción y demolición, árido reciclado, mezclas bituminosas.

\section{INTRODUCCIÓN}

En la actualidad está extendida la idea de la necesidad de un desarrollo sostenible que gestione de forma correcta los bienes escasos que el medio aporta. Ante este reto, la industria de la construcción ha sido pionera en el desarrollo de técnicas para la reutilización de los materiales de desecho que genera. La Unión Europea (UE) fomenta una política de reciclado a través de una serie de investigaciones de carácter técnico para poder dar uso a estos materiales. En este sentido, hay todo un catálogo de residuos que contempla la UE y que se conoce como CER (Catálogo Europeo de Residuos), donde los residuos de construcción y demolición (RCD) se contemplan con el código CER 170000. Los RCD se conocen habitualmente como "escombros". Se generan en grandes cantidades y su volumen supera al de origen doméstico. Su poder contaminante es bastante bajo. Por el contrario, su impacto visual es con frecuencia alto, debido tanto al gran volumen que ocupan como al escaso control ambiental ejercido sobre los terrenos elegidos para su depósito. Un segundo impacto ecológico negativo se deriva del despilfarro de materias primas. Por estas razones en España se aprobó el Plan Nacional de Residuos de Construcción y Demolición (PNRCD) [1] estableciendo como uno de sus objetivos el reciclaje del 60\% de los RCD en el año 2006. Sin embargo, este porcentaje en España es inferior al 5\%, existiendo una red insuficiente de plantas de reciclaje. En Europa la media de este porcentaje se sitúa en torno al $28 \%$.

En este orden de ideas cabe decir que dada la enorme cantidad de áridos que se consumen en los firmes de carreteras, parece adecuado pensar en la posibilidad del uso de los RCD en sus diversas capas. En este sentido, en la actualidad existe diversa literatura técnica sobre la caracterización de hormigones estructurales elaborados con áridos reciclados de RCD [2,3]. Asimismo, existen experiencias sobre el empleo de estos materiales en carreteras como capas granulares y capas tratadas con conglomerantes hidráulicos $[4,5,6]$. Sin embargo, los autores de este artículo no conocen antecedentes de estudios técnicos de caracterización de estos residuos para su empleo como mezclas bituminosas para capas de firmes de carreteras. Por lo dicho anteriormente, el objetivo de esta investigación es ampliar el uso de los áridos reciclados más allá de una simple zahorra para una capa granular o de un material tipo grava-cemento. En concreto, se abordará el estudio de las propiedades mecánicas de las mezclas bituminosas en caliente elaboradas con áridos reciclados para su empleo en carreteras.

\section{PARTE EXPERIMENTAL}

Se investigó la factibilidad de dosificar dos tipos de mezclas bituminosas en caliente a partir de áridos reciclados (AR) en diferentes proporciones. Asimismo, con el fin de comparar los resultados se dosificaron los mismos tipos de mezclas bituminosas elaboradas con áridos naturales (AN) de cantera. Las dosificaciones de las mezclas se hicieron de acuerdo al Pliego de Prescripciones Técnicas Generales para Obras de Carreteras y Puentes, también denominado PG-3 [7]. 


\subsection{Definición de las mezclas}

Se estudió como capa de base una mezcla G-20; mientras que como capa intermedia se estudió una mezcla S20. La mezcla G-20 es una mezcla gruesa cuya granulometría es continua, con un tamaño máximo de $25 \mathrm{~mm}$. Está compuesta en casi tres cuartas partes por árido grueso, con un contenido de filler y betún que ronda el 4 $\%$. La mezcla S-20 es una mezcla semidensa de granulometría continua, con tamaño máximo de $25 \mathrm{~mm}$. Está compuesta en torno a las dos terceras partes por árido grueso, con un contenido de filler y betún que ronda el $5 \%$. En la Tabla 1 se presentan los husos granulométricos de estas dos mezclas bituminosas.

Tabla 1. Husos y curvas y granulométricas

\begin{tabular}{|c|c|c|c|c|c|c|c|c|c|c|c|c|}
\hline \multirow{3}{*}{\multicolumn{2}{|c|}{ Mezcla }} & \multicolumn{11}{|c|}{ Tamaños de los tamice UNE-EN-933-2 (mm.) } \\
\hline & & \multicolumn{6}{|c|}{ Árido grueso } & \multicolumn{4}{|c|}{ Árido fino } & \multirow{2}{*}{$\begin{array}{l}\text { Filler } \\
0.063 \\
\end{array}$} \\
\hline & & 40 & 25 & 20 & 12.5 & 8 & 4 & 2 & 0.500 & 0.250 & 0.125 & \\
\hline \multirow{3}{*}{$\begin{array}{c}\text { Semidensa } \\
\text { S-20 }\end{array}$} & Huso & --- & 100 & $80-95$ & $64-79$ & $50-66$ & $35-50$ & $24-38$ & $11-21$ & $7-15$ & $5-10$ & $3-7$ \\
\hline & AN & --- & 100 & 95 & 78 & 63 & 40 & 30 & 15 & 12 & 8 & 5.5 \\
\hline & AR & --- & 100 & 95 & 78 & 63 & 40 & 30 & 15 & 12 & 8 & 6.5 \\
\hline \multirow{3}{*}{$\begin{array}{c}\text { Gruesa } \\
\text { G-20 }\end{array}$} & Huso & --- & 100 & $75-95$ & $55-75$ & $40-60$ & $25-42$ & $18-32$ & $7-18$ & $4-12$ & $3-8$ & $2-5$ \\
\hline & AN & --- & 100 & 95 & 75 & 60 & 37.75 & 28.5 & 13 & 10 & 7 & 4.25 \\
\hline & AR & --- & 100 & 95 & 75 & 60 & 37.75 & 28.5 & 13 & 10 & 7 & 4.75 \\
\hline
\end{tabular}

Para caracterizar los materiales básicos se utilizaron las normas técnicas españolas NLT [8] (Ensayos de Carreteras) del Centro de Estudio de Carreteras y las normas UNE-EN [9] de la Asociación Española de Normalización y Certificación (AENOR).

Betún. Se optó por elegir un betún de uso convencional con propiedades medias. En concreto se seleccionó un betún de penetración 60/70 (NLT-124), con un valor del punto de reblandecimiento de anillo y bola (NLT-125) igual a 48.5. El índice de penetración Pleiffer (NLT-181) es igual a -0.8. La densidad es de 1.03 $\mathrm{g} / \mathrm{cm}^{3}$ (NLT-122). Este betún posibilita su uso en multitud de circunstancias de tráfico y climas. En una zona templada se puede utilizar como capa de rodadura, intermedia y de base. Se comprobó que el betún cumple todas las especificaciones del PG-3.

Áridos naturales. Estos áridos fueron proporcionados por una cantera en fracciones 0/6, 6/12 y 12/25. La roca de la que proceden los áridos es un esquisto feldespático de grano fino producido por metamorfismo regional, compuesto por filitas grises con abundantes lentes de cuarzo microscópicas intercaladas entre unos planos de esquistosidad muy plegados. Sus componentes principales son cuarzo (35\%), feldespato sódico $(30 \%)$, mica incolora o moscovita $(20 \%)$ y clorita $(15 \%)$. Esta roca presenta un color gris lustroso y buenas propiedades como árido para capas de base, intermedia y de rodadura. En la tabla 2 se reflejan las características principales de los áridos correspondientes a las granulometrías S-20 y G-20. Como se observa, cumple las especificaciones del PG-3, ya que el índice de lajas en todas las fracciones ensayadas es $<20 \%$ (UNE-EN933-3), todas sus caras son de fractura (UNE-EN933-5); el equivalente de arena es de un 70.1 y un 75.4\% (UNE-EN933-8); el coeficiente de Los Ángeles está comprendido es 19.4 y 22.1\% (UNE-EN1097-2). El coeficiente de absorción del agua es 0.17 y 0.19\% (UNE-EN-1097-6). La densidad de los áridos en aceite de parafina (NLT-167) es $2.69 \mathrm{~g} / \mathrm{cm}^{3}$.

Tabla 2. Características de los áridos

\begin{tabular}{|l|c|c|c|c|c|}
\hline \multirow{2}{*}{ Ensayo } & \multicolumn{2}{|c|}{$\mathbf{1 0 0 \%}$ AN } & \multicolumn{1}{c|}{$\mathbf{1 0 0 \%}$ AR } & \multicolumn{2}{c|}{$\mathbf{5 0 \%}$ AR + 50 \%AN } \\
\cline { 2 - 6 } & $\mathbf{S - 2 0}$ & $\mathbf{G - 2 0}$ & $\mathbf{S - 2 0}$ & $\mathbf{S - 2 0}$ & G-20 \\
\hline Índice de lajas (\%) & 17.2 & 19.4 & 16.2 & 17.1 & 18.3 \\
\hline Caras de fractura (\%) & 100 & 100 & 100 & 100 & 100 \\
\hline Equivalente de arena (\%) & 75.4 & 70.1 & 87.2 & 85.4 & 88.1 \\
\hline Coeficiente de los Ángeles (\%) & 19.4 & 22.1 & 39.65 & 25.6 & 24.8 \\
\hline Coeficiente de absorción de agua (\%) & 0.17 & 0.19 & 6.1 & 4.59 & 4.82 \\
\hline Densidad relativa en aceite de parafina $\left(\mathrm{g} / \mathrm{cm}^{3}\right)$ & 2.69 & 2.69 & 2.58 & 2.66 & 2.66 \\
\hline
\end{tabular}

Áridos Reciclados. Los áridos reciclados tienen la granulometría requerida para rellenos en la fracción 0/40. Su composición en peso es la siguiente: hormigón (72\%); Piedra (20\%); Cerámico (2\%) y betún (6\%). En la tabla 2 se observan sus características principales. Cuando, como se explica en el apartado 3.1, en la granulometría S-20 se utiliza un $100 \%$ de AR los valores del índice de lajas, caras de fractura y equivalente de arena cumplen los requisitos del PG-3. Sin embargo, el coeficiente de desgaste de Los Ángeles supera 
ampliamente los valores especificados, ya que presenta un resultado del 39.65\%. También, cabe destacar el alto valor del coeficiente de absorción del agua, igual al 6.1\%, 36 veces superior al valor de la granulometría S-20 con un $100 \%$ de AN. La densidad de los áridos en aceite de parafina es $2.58 \mathrm{~g} / \mathrm{cm}^{3}$. Por otra parte, al emplear en todos las fracciones, tanto de las granulometrías S-20 como G-20, un 50\% de AN + un 50\% de AR se observó que los valores del índice de lajas, del equivalente de arena también cumplen los valores establecidos. En este caso, lógicamente, se obtuvieron unos menores valores de los coeficientes de Los Ángeles, aunque todavía no cumplen lo especificado en el PG-3 para carreteras con tráfico medio. Los valores del coeficiente de absorción del agua siguen siendo elevados, del orden de 27 a 25 veces los valores obtenidos con un $100 \%$ de AN. Por último, al combinar los dos tipos de áridos se produce un incremento de la densidad relativa en aceite de parafina hasta alcanzar un valor igual a $2.66 \mathrm{~g} / \mathrm{cm}^{3}$.

Filler. El filler utilizado en todos los casos fue el proveniente del proceso de trituración del árido natural. Esto se hizo así procurando que el mástico se comporte de igual manera en los dos casos.

\subsection{Ensayos mecánicos}

A continuación se presentan los ensayos seleccionados para la caracterización mecánica de las mezclas. Los ensayos Marshall, inmersión-compresión y de pista en laboratorio se realizaron con objeto de buscar la dosificación óptima de la mezclas para poder ser utilizada dentro de alguna de las categorías de tráfico recogidas en el PG-3, Tabla 3. Como complemento de estos estudios se realizaron ensayos de fatiga, que sin ser preceptivos en el Pliego de carreteras, permiten profundizar en el comportamiento de la mezcla bituminosa frente al inicio de fisuras debido a la presencia de solicitaciones cíclicas.

Tabla 3. Criterios de dosificación preceptivos en el PG-3

\begin{tabular}{|c|c|c|c|c|c|c|}
\hline \multirow{2}{*}{\multicolumn{3}{|c|}{ Característica }} & \multicolumn{4}{|c|}{ Categoría de tráfico pesado $^{+}$} \\
\hline & & & T00 y T0 & $\mathrm{T} 1$ y T2 & T3 y arcenes & $\mathrm{T} 4$ \\
\hline \multirow{5}{*}{ 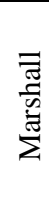 } & \multicolumn{2}{|l|}{ Estabilidad $(\mathrm{kN})$} & $>15$ & $>12.5$ & $>10$ & $8-12$ \\
\hline & \multicolumn{2}{|l|}{ Deformación (mm.) } & $2-3$ & \multicolumn{2}{|r|}{$2-3.5$} & $2.5-3.5$ \\
\hline & \multirow[t]{2}{*}{ Huecos en mezcla $(\%)$} & Capa intermedia S-20 & $4-6$ & $5-8$ & $4-8$ & $4-8^{*}$ \\
\hline & & Capa de base G-20 & $5-8$ & $6-9$ & $5-9$ & --- \\
\hline & \multicolumn{2}{|l|}{ Huecos en áridos (\%) } & \multicolumn{4}{|c|}{$>14$} \\
\hline \multicolumn{3}{|c|}{ Índice de resistencia conservada IC (\%) } & \multicolumn{4}{|c|}{$>75$} \\
\hline \multirow{2}{*}{\multicolumn{2}{|c|}{$\begin{array}{l}\text { Velocidad de deformación VD } \\
\qquad(\mu \mathrm{m} / \mathrm{min})\end{array}$}} & Capa intermedia S-20 & \multicolumn{4}{|c|}{$<20$} \\
\hline & & Capa de base G-20 & \multicolumn{4}{|c|}{$-\overline{---}$} \\
\hline
\end{tabular}

Marshall (NLT-159). Se compactaron cinco series de tres probetas cilíndricas, de $101.6 \mathrm{~mm}$ de diámetro por $63.5 \mathrm{~mm}$ de alto. Cada serie se fabricó con idéntica granulometría pero distinto porcentaje de betún sobre el peso de árido. Para cada porcentaje de betún se calculó la media de los siguiente parámetros: Huecos en áridos, Ha (\%); Huecos en mezcla, Hm (\%); Densidad aparente, Da $\left(\mathrm{g} / \mathrm{cm}^{3}\right)$; Deformación, D (mm) y Estabilidad, E $(\mathrm{kN})$. Los resultados de este ensayo se utilizaron para definir el porcentaje de óptimo de betún (Bo) de las mezclas para cumplir con los criterios establecidos en el PG-3, Tabla 3. Las probetas que se fabricaron para el resto de los ensayos se dosificaron con el porcentaje de betún óptimo seleccionado en este ensayo.

Efecto del agua en la resistencia de las mezclas bituminosas (NLT-161 y NLT-162). Se fabricaron 10 probetas cilíndricas de dimensiones: $101.6 \mathrm{~mm}$ de diámetro y $101.6 \mathrm{~mm}$ de alto. Cinco probetas se sumergieron en un baño de agua a $60^{\circ} \mathrm{C}$ durante 24 horas y cinco no. Posteriormente, se sometieron a compresión simple y se determinó la media de la tensión máxima alcanzada en cada grupo. El Índice de Resistencia Conservada IC $(\%)$, se calcula dividiendo la resistencia del grupo que estuvo inmerso a $60{ }^{\circ} \mathrm{C}$ entre la resistencia del grupo que no estuvo. Mediante enste ensayo se caracteriza la pérdida de cohesión que se produce por la acción del agua en las mezclas. Según el PG-3 los valores de IC en mezclas S-20 y G-20 debe ser superior al $75 \%$ (Tabla 3 ).

Pista en laboratorio (NLT-173). Se fabricaron tres probetas prismáticas de dimensiones $300 \times 300 \times 50$ $\mathrm{mm}$. El ensayo consiste en someter una probeta al paso alternativo de una rueda en condiciones determinadas de presión y temperatura, midiéndose periódicamente la profundidad de la deformación producida. La media de la velocidad de deformación (VD) se midió en el intervalo de tiempo comprendido entre los 105 a los 120 
minutos desde el comienzo. Este ensayo solamente es obligatorio para capas de rodadura o intermedias (tabla 3).

Fatiga a flexotracción dinámica (NLT-350). Se fabricaron diez probetas prismáticas de dimensiones 300 × 50 × $50 \mathrm{~mm}$ y se ensayaron en flexotracción en una prensa servohidráulica en control de desplazamiento a una frecuencia de $10 \mathrm{~Hz}$. Los valores de deformación se midieron mediante un extensómetro dinámico colocado en la parte inferior de la probeta que es donde se soportan las mayores tensiones de tracción. A partir de estos ensayos se determinó para cada mezcla; la ley de fatiga, el módulo dinámico y el ángulo de desfase.

Mediante la ley de fatiga se cuantifica el agotamiento del material provocado por el daño acumulado ante la presencia de solicitaciones cíclicas y, que se manifiesta en la fisuración del mismo. La ley de fatiga relaciona el número de ciclos de carga hasta la fatiga del material $(\mathrm{N})$, con el nivel de deformación $(\varepsilon)$ producida por la solicitación cíclica. $\varepsilon$ y $\mathrm{N}$ están ligados por la expresión:

$\varepsilon=k N^{B}$

donde $\mathrm{k}$ y $\mathrm{B}$ son constantes que describen el comportamiento a fatiga del material y dependen entre otros factores de los áridos de la mezcla.

El módulo dinámico (MD) es una medida de la rigidez de la mezcla y se calcula como el cociente entre la amplitud cíclica de la función tensión $\left(T_{c}\right)$ y la amplitud cíclica de la función deformación $\left(\varepsilon_{c}\right)$ en el ciclo 200. Su expresión es la siguiente:

$M D=T_{c} / \varepsilon_{c}$.

El ángulo de desfase $(\varphi)$ da una idea del predominio del carácter elástico o viscoso de la mezcla estudiada, un ángulo de $\varphi=0^{\circ}$ indicaría que el material es elástico, mientras que un ángulo de $\varphi=90^{\circ}$ indicaría que el material es viscoso. Se calcula mediante la expresión:

$\varphi=\left(B_{t}-B_{\varepsilon}\right) 180 / \pi$

donde $B_{t}$ y $B_{\varepsilon}$ son los ángulos de fase de las funciones tensión y deformación.

\section{RESULTADOS Y DISCUSIÓN}

\subsection{Resultados de los ensayos Marshall}

Los resultados obtenidos con el método Marshall evidenciaron la imposibilidad de utilizar únicamente árido reciclado en mezcla. Para la mezcla S-20-AR, compuesta por $100 \%$ árido reciclado, se observó que las mezclas tenían poca cohesión, el árido más grueso no se encontraba correctamente envuelto por el betún y, el conjunto tenía un aspecto terroso. Este comportamiento se debe a la excesiva porosidad del árido; al aumentar la superficie específica del mismo se requieren mayores cantidades de betún para conseguir la suficiente cohesión. Las mezclas fabricadas con un 5,90\% de betún, arrojaron valores de Ha, E y D elevados, pero dentro del PG-3. En cambio, el valor de Hm era del 11.7 (\%), por tanto, no se cumplió este criterio preceptivo para la fabricación de mezclas bituminosas en caliente para su uso en firmes. Para las dosificaciones realizadas con menores cantidades de betún no se consiguió consistencia en las mezclas, que terminaban por disgregarse.

Del análisis de los resultados anteriores se optó por dosificar las mezclas con AR utilizando un $50 \%$ de árido reciclado. En las mezclas con AN se eligió la cantidad de filler en función de la relación ponderal filler-betún recomendada en el PG-3; mientras que en las mezclas con AR se tuvo que elevar la cantidad de filler hasta casi el límite superior del huso. En la figura 1 se representan de forma gráfica los resultados de los ensayos Marshall, tanto de las mezclas AN como de las AR. A este respecto, se pueden hacer los comentarios siguientes:

1. Las curvas de Ha no presentan grandes pendientes, siendo poco susceptibles al contenido del betún. Las mezclas con AR producen un aumento de los Ha de entre el $3 \%$ y el $5 \%$ con respecto a las mezcla con AN. La causa está en que la mayor absorción de betún por parte de los áridos reciclados disminuye el grosor de la película que envuelve los áridos y en definitiva disminuye la facilidad de encaje entre los mismos. 
2. Las curvas de $\mathrm{Hm}$ son descendentes con el contenido de betún hasta alcanzar un mínimo donde aproximadamente se mantiene constante. El porcentaje de betún utilizado no fue lo suficientemente elevado para alcanzar este mínimo. Las mezclas con AR presentan un $3.0 \%$ más de Hm para la mezcla G-20 y un 7.0 \% más para la mezcla S-20. Según estos resultados se puede predecir un comportamiento peor de las mezclas con AR frente a la entrada de agua.

3. La curva de deformación es creciente con el contenido de betún. Siendo similar en todos los casos.

4. Se aprecian las ramas ascendentes y descendentes de la estabilidad tanto para la mezcla S-20-AN como para la mezcla G-20-AR. La mezcla G-20-AN presenta solamente la rama ascendente; mientras que la mezcla S-20-AR presenta únicamente la rama descendente.

5. Las densidades de las mezclas con AR son ligeramente inferiores a las de las mezclas con AN. Esto se debe a la mayor dificultad de compactación de las mezclas con AR.
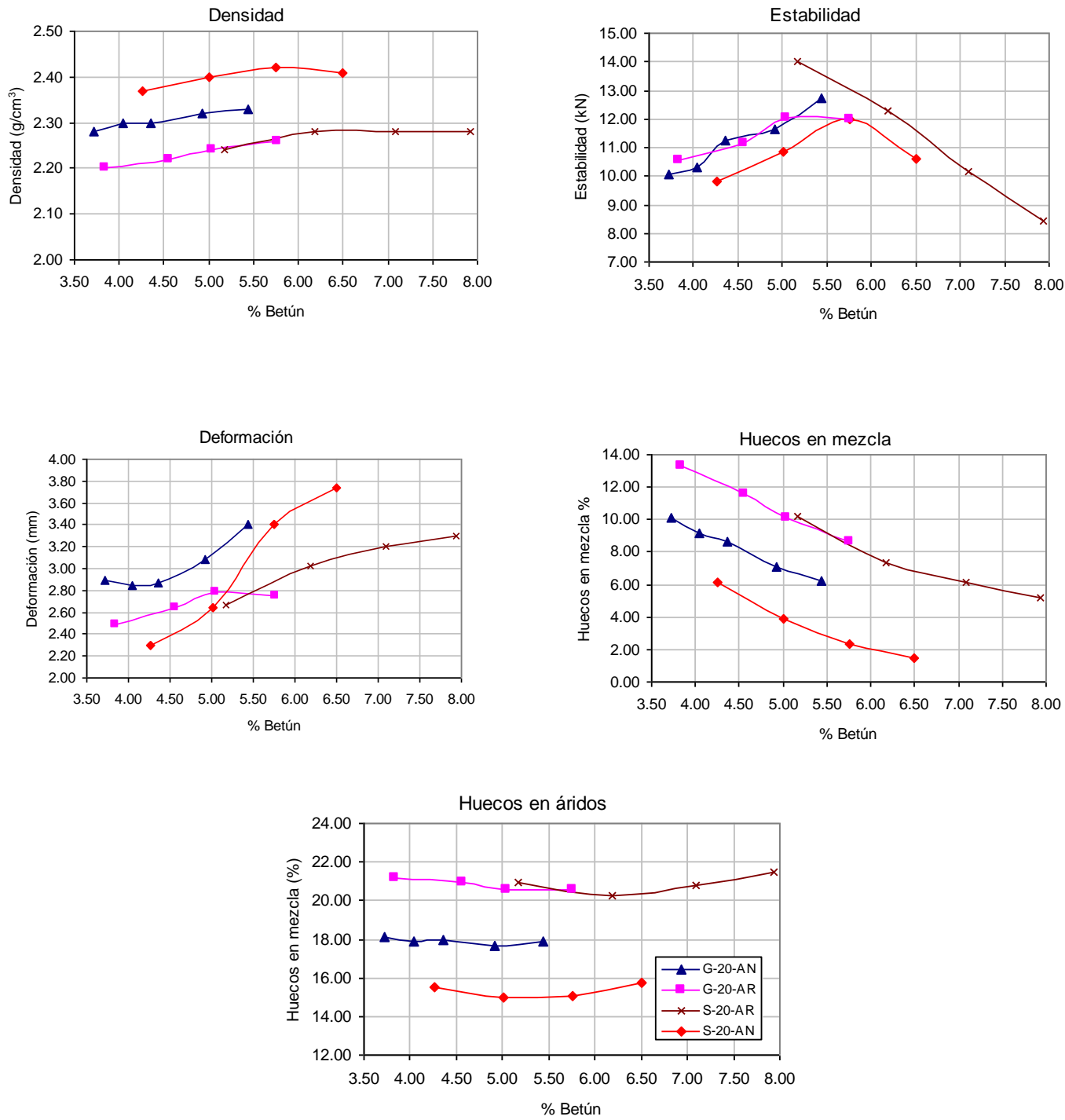

Figura 1. Ensayo Marshall

A partir de los resultados obtenidos del ensayo Marshall se seleccionaron las proporciones de $\mathrm{B}_{\mathrm{o}}$, para cada tipo de mezcla, que cumpliera con los requisitos del PG-3 para las diferentes categorías de tráfico (Tabla 3). En la mezcla S-20-AN el porcentaje de Bo adoptado es igual al 5\% sobre el peso total de los áridos. Con esta dosificación la mezcla cumple para las categorías de tráfico T3 y T4. Para la mezcla G-20-AN el porcentaje de Bo adoptado es igual al $4.25 \%$, por tanto, se verifican todos los requisitos de los T3 y T4. En la mezcla S20-AR el porcentaje de Bo es el 5.90\%, confirmándose todos los requisitos desde T1 hasta T4. Finalmente, en la mezcla G-20-AR el porcentaje de Bo es igual al 4.75\%; aquí el comportamiento es peor, ya que 
solamente se cumplen todos los requisitos de la categoría T4. Los resultados del ensayo Marshall para las dosificaciones seleccionadas se incluyen en la Tabla 4.

\section{2. Índice de resistencia conservada}

Los resultados del índice de resistencia conservada IC(\%) se presentan en la Tabla 4. Para las mezclas S-20AN y G-20-AN los valores obtenidos son ligeramente superiores del $75 \%$ marcado en la Tabla 3 . Con respecto a las mezclas con AR la resistencia conservada no cumple con las especificaciones de la Tabla 3. En particular los valores de la G-20 AR fueron inferiores a los obtenidos con las mezclas S-20-AR

La mezcla S-20-AR presenta un valor de huecos en mezcla no excesivamente alto (8\%). Por tanto, la entrada de agua en el interior de la misma y la consiguiente pérdida de resistencia debería ser parecida al de otras mezclas con la misma cantidad de huecos. Una posible explicación a este resultado sería que dada la naturaleza de los áridos reciclados, que contiene entre ellos materiales arcillosos y hormigón, podría haber favorecido la retención de agua en su interior, desplazando el betún y disminuyendo consecuentemente la resistencia. Los valores tan bajos obtenidos para la G-20-AR se pueden explicar en base al elevado porcentaje de huecos en mezcla que presenta sumado a la naturaleza arcillosa de los RCD (igual que en la mezcla S-20-AR).

\subsection{Deformación permanente}

Los resultados de la media de las VD de las probetas de las mezclas S-20 se presentan en la Tabla 4. En la mezcla S-20-AN, la velocidad VD es igual a $19.29 \mu \mathrm{m} / \mathrm{min}$; y en la mezcla S-20-AR es $15.07 \mu \mathrm{m} / \mathrm{min}$. Ambas cumplen el límite de $20 \mu \mathrm{m} / \mathrm{min}$., (Tabla 3). Las mezclas G-20 no fueron ensayadas por que no es preceptivo en el PG-3. En la figura 2 se representan las curvas deformación-tiempo de las dos mezclas. Obsérvese que la mezcla S-20-AN presenta una elevada deformación al principio del ensayo, pero a medida que éste discurre se va estabilizando. En cambio, en la mezcla S-20-AR, al principio del ensayo la deformación es menos elevada que en el caso anterior. En la mezcla S-20-AN la estabilización de VD se produce antes que en la S-20-AR. Hacia el final del ensayo la deformación se va incrementando ligeramente en la S-20-AN, mientras que van decreciendo en la S-20-AR. De no variar esta tendencia, la S-20-AN podría ser poco resistente a largo plazo a las deformaciones plásticas. La mezcla S-20-AR se comporta mejor frente a las deformaciones plásticas.

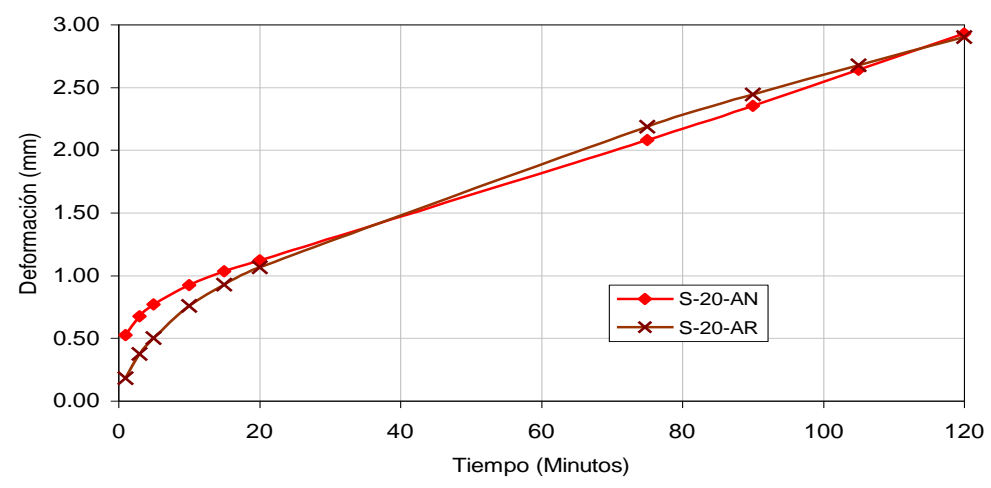

Figura 2. Ensayo de Pista de Laboratorio

\subsection{Ley de fatiga}

Los valores experimentales de $\varepsilon$ y $\mathrm{N}$ para las mezclas estudiadas están representados en escala logarítmica en las figuras $3 \mathrm{a}$ y $3 \mathrm{~b}$. En estas figuras se han incluido también las leyes de fatiga del Centro de Experimentación de Obras Públicas (Cedex) [10] y las contempladas en la Instrucción 6.1-IC [11] para las mezclas G-20 y S-20 para comparar los resultados obtenidos y determinar si el agotamiento que sufren las mezclas ante la presencia de cargas cíclicas está dentro de los límites esperados.

En la figura 3 a se observa que tanto la mezcla G-20-AN como la mezcla G-20-AR presentan un comportamiento a fatiga muy similar. Esto se refleja en que sus pendientes $(B)$ y coeficientes $(k)$ son similares (Tabla 4). Con respecto a las curvas del Cedex y de la Instrucción, las mezclas G-20-AN y G-20AR, por un lado están situadas sobre la ley Cedex 2; y, por otro lado, están siempre por debajo de la ley de la instrucción (con pendientes ligeramente más pronunciadas).

En la figura $3 \mathrm{~b}$ se puede observar que la mezcla $S-20$-AN y la mezcla S-20-AR presentan un comportamiento a fatiga diferente. La mezcla S-20-AR tiene una pendiente $(B)$ mucho más pronunciada que la mezcla $\mathrm{S}-20$ - 
AN. La ley de la mezcla S-20-AR está dentro de ese intervalo marcado por el CEDEX (con pendiente más pronunciada) y, por el contrario, la ley de la mezcla S-20-AN se sale favorablemente fuera del mismo (con pendiente mucho menos pronunciada). La pendiente de la ley de la instrucción es más pronunciada que la ley de de la mezcla S-20-AN. Para una misma deformación unitaria la ley de la mezcla S-20-AN predice un mayor número de ciclos para llegar al agotamiento que las leyes de la mezcla S-20-AR y de la instrucción. Se puede deducir que las mezclas S-20-AN presentan un mejor comportamiento que las mezclas S-20-AR, aunque el de ésta última es aceptable.

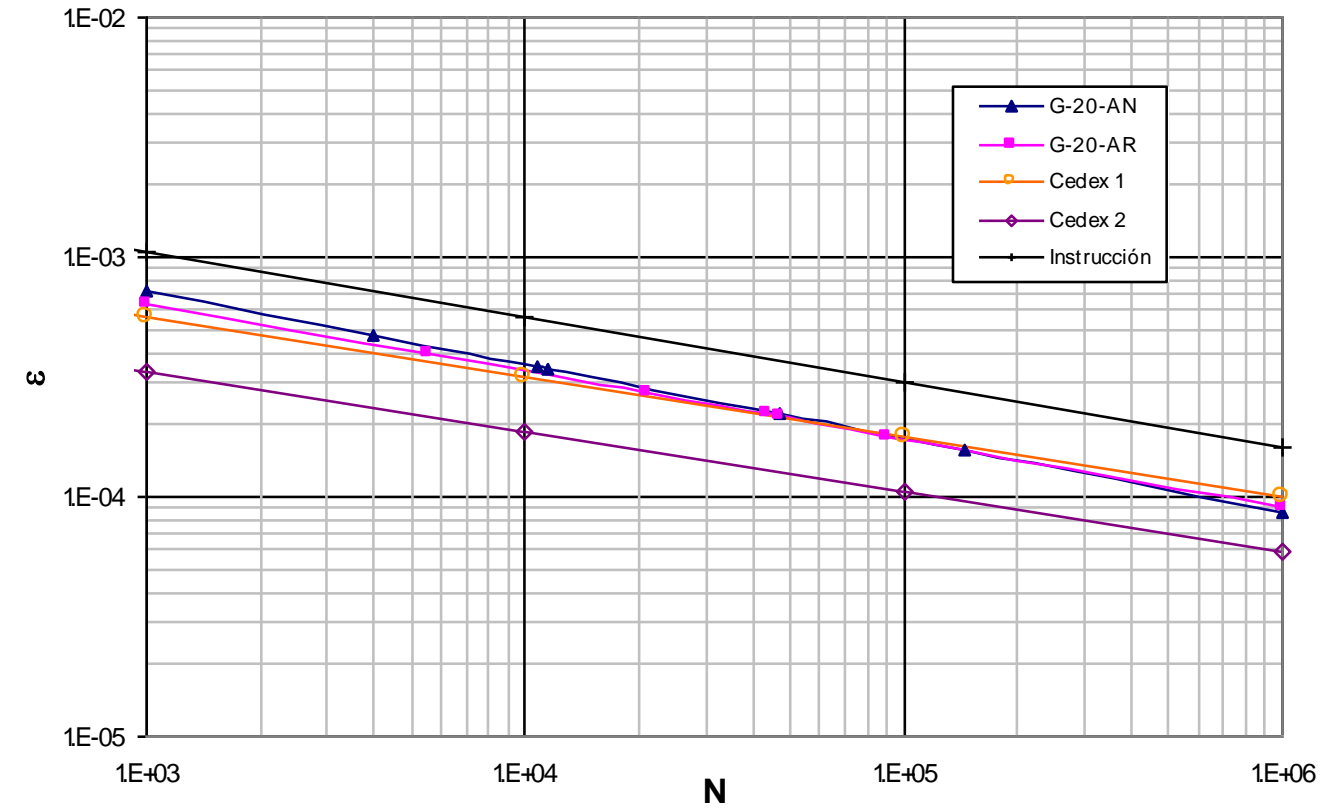

Figura 3a. Leyes de fatiga de las mezclas G-20

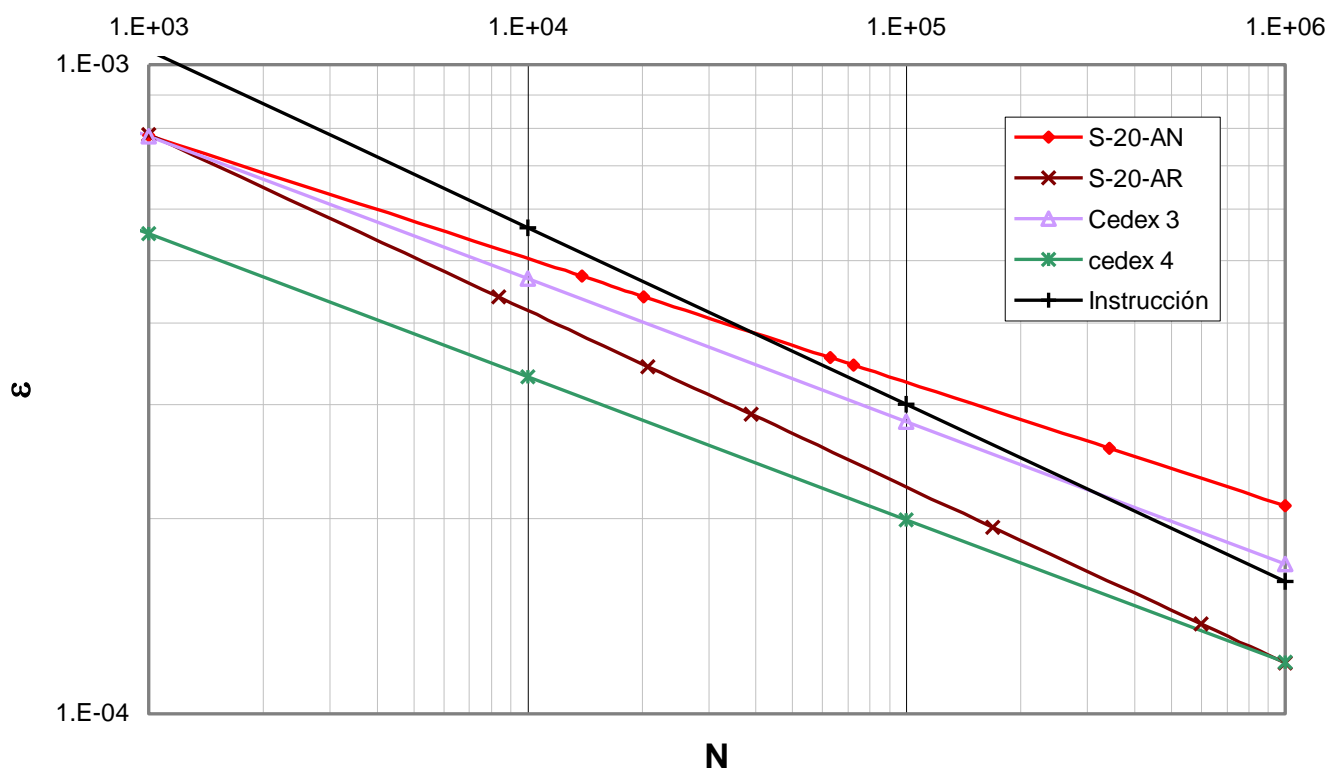

Figura 3b. Leyes de fatiga de las mezclas S-20

\section{Módulo Dinámico}

Los valores de los módulos dinámicos se presentan en la Tabla 4. Los valores del módulo dinámico de las

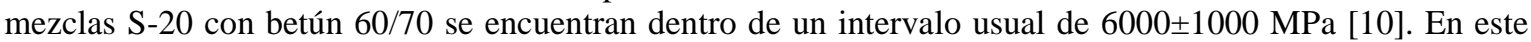
sentido la mezcla S-20-AN es ligeramente inferior al módulo usual para este tipo de mezclas. Sin embargo, la mezcla S-20-AR está dentro de ese intervalo. Por otra parte, el intervalo de valores de los módulos para las mezclas G-20 con un betún 60/70 es de 5500ะ1000 MPa. En este sentido la mezcla G-20-AN está por debajo de ese intervalo y la mezcla G-20-AR está dentro del mismo. Se obtienen mezclas de mayor rigidez cuando 
se añade AR. Las rigideces concuerdan de manera razonable con la capacidad de soportar las cargas de las mezclas, reflejado en los valores de estabilidad obtenidos en los ensayos Marshall (figura 1). Estos módulos bajos ponen de manifiesto un punto de debilidad de las mezclas fabricadas con AN. Al no ser lo suficientemente rígidas, con el paso del tráfico, se deformarían de forma excesiva al no absorber de forma adecuada las tensiones. Ello podría favorecer una fisuración temprana de la mezcla más superficial al tener una capa de base poco rígida.

\section{Ángulo de desfase}

En la Tabla 4 se exponen los resultados del ajuste de las funciones tensión y deformación en el ciclo 200. Estos valores de desfase se encuentran dentro de lo normal, resultando muy parecidos a los obtenidos por otros autores [12]. La mezcla S-20-AR es la más elástica (tiene además el mayor módulo); mientras que la mezcla S-20-AN (con el menor módulo de todas) es la más viscosa.

Tabla 4. Resultados de los ensayos

\begin{tabular}{|c|c|c|c|c|c|c|c|c|c|c|c|c|c|}
\hline \multirow[b]{2}{*}{ Mezcla } & \multicolumn{6}{|c|}{ Ensayo Marshall } & \multirow[b]{2}{*}{$\begin{array}{l}\mathrm{IC} \\
(\%)\end{array}$} & \multirow[b]{2}{*}{$\begin{array}{c}\text { VD } \\
(\mu \mathrm{m} / \mathrm{min})\end{array}$} & \multicolumn{3}{|c|}{ Ley de fatiga } & \multirow[b]{2}{*}{$\begin{array}{c}\mathrm{MD} \\
(\mathrm{MPa})\end{array}$} & \multirow[b]{2}{*}{$\varphi$} \\
\hline & $\begin{array}{l}\text { Bo } \\
(\%)\end{array}$ & $\begin{array}{l}\mathrm{Ha} \\
(\%)\end{array}$ & $\begin{array}{l}\mathrm{Hm} \\
(\%)\end{array}$ & $\begin{array}{c}\mathrm{D} \\
(\mathrm{mm})\end{array}$ & $\begin{array}{c}\mathrm{E} \\
(\mathrm{kN})\end{array}$ & $\begin{array}{c}\mathrm{Da} \\
\left(\mathrm{g} / \mathrm{cm}^{3}\right)\end{array}$ & & & $k$ & $B$ & $\mathrm{R}^{2}$ & & \\
\hline S-20-AN & 5.00 & 15 & 4 & 2.6 & 10.8 & 2.4 & 76.99 & 19.29 & 0.0029 & -0.1903 & 0.8575 & 3866 & 45.85 \\
\hline S-20-AR & 5.90 & 20.2 & 8 & 2.9 & 12.6 & 2.28 & 63.72 & 15.07 & 0.0051 & -0.2716 & 0.9697 & 5016 & 35.05 \\
\hline G-20-AN & 4.25 & 18 & 8.9 & 2.8 & 11 & 2.3 & 79.34 & ---- & 0.0060 & -0.3064 & 0.8620 & 4086 & 37.71 \\
\hline G-20-AR & 4.75 & 20.9 & 11 & 2.7 & 11.5 & 2.22 & 50.26 & ---- & 0.0045 & -0.2823 & 0.9502 & 4675 & 37.05 \\
\hline
\end{tabular}

\section{CONCLUSIONES}

Las principales conclusiones que se pueden extraer de este trabajo de investigación son las siguientes:

1) Se ha podido llevar a cabo una dosificación de mezclas bituminosas que contienen árido reciclado en un $50 \%$ de peso, en todas las fracciones. Estas mezclas tienen un contenido de betún superior al de las mezclas realizadas sólo con árido natural y además necesitan una mayor cantidad de filler, y sólo así, se han podido cumplir los requisitos que el PG-3 establece para los parámetros obtenidos en el ensayo Marshall en carreteras de bajo tráfico. Por otro lado, no se ha podido realizar una dosificación de mezclas realizadas únicamente con árido reciclado.

2) Las mezclas realizadas con árido natural presentan un comportamiento adecuado a la acción del agua, cumpliendo los requisitos establecidos para el ensayo de inmersión-compresión, mientras que las mezclas realizadas con un 50\% de árido reciclado presentan una pérdida de resistencia excesiva.

3) En general las mezclas S-20 se comportan bien frente a las deformaciones plásticas, cumpliendo con los valores exigidos en el PG-3 para carreteras de bajo tráfico.

4) En cuanto a las leyes de fatiga obtenidas, éstas son muy parecidas a las de otros autores. Los valores del módulo dinámico son razonables, siendo mayores en mezclas con árido reciclado al 50\%.

5) De acuerdo a las conclusiones anteriores se puede decir que existe la posibilidad de uso de mezclas realizadas con árido reciclado en carreteras de bajo tráfico. No obstante, todavía hay que seguir investigando en las distintas soluciones que se pueden introducir para mejorar el comportamiento frente al agua. Así, se presenta una serie de alternativas para alcanzar resultados más favorables en el futuro; entre las que se destacan:

- Selección de áridos reciclados de mejor calidad

- Disminución de la proporción de árido reciclado en la mezcla

- Utilización de fílleres de aportación como cal y cemento

- Utilización de betunes más duros o modificados

\section{BIBLIOGRAFÍA}

[1] Ministerio de Medio Ambiente: Plan Nacional de Residuos de Construcción y Demolición 2001-2006. BOE no 166 (2001). pp. 25305-25313. 
[2] M. Sánchez de Juan: Estudio sobre la utilización de áridos reciclados para la fabricación de hormigón estructural. p. 505. Tesis doctoral. Universidad Politécnica de Madrid. Escuela Técnica Superior de Ingenieros de Caminos, Canales y Puertos. Madrid, 2004.

[3] B. González, F. Martínez: Hormigones con áridos reciclados: estudio de propiedades de los áridos y de las mezclas. Materiales de Construcción. Vol. 55, no 279 (2005), pp. 53-66.

[4] T. Park: Application of Construction and Building Debris as Base and Subbase Materials in Rigid Pavement. Journal of Transportation Engineering, Vol. 129, $\mathrm{n}^{\circ} 5$ (2003), pp-558-563.

[5] T. Bennert, W. Papp, A. Maher, N. Gucunski: Utilization of construction and Demolition Debris Under Traffic-Type Loading in Base and Subbase Applications. Transportation Research Record, $\mathrm{n}^{\circ} 1714$ (2000), pp.33-39.

[6] Asociación Española de Normalización y Certificación (AENOR). UNE146131, Áridos para capas granulares y capas tratadas con conglomerantes hidráulicos para su uso en capas estructurales de firmes, Anexo A (informativo), Áridos reciclados. Madrid, 2003.

[7] Dirección General de Carreteras. Pliego de prescripciones técnicas generales para obras de carreteras y puentes PG-3. Ministerio de Fomento. Ediciones Liteam, Madrid, 2002.

[8] Dirección general de Carreteras. Normas NLT. Ensayos de carreteras. Ministerio de Obras Públicas y Transportes (MOPT), $2^{\text {a }}$ Edición, Madrid, 1992.

[9] Asociación Española de Normalización y Certificación (AENOR). Manual de normas técnicas UNE-EN. Serie construcción. Áridos para mezclas bituminosas y tratamientos superficiales de carreteras. p. 526. Madrid, 2001.

[10] J. García. Caracterización de módulos, fatiga y otros ensayos dinámicos. Jornada sobre caracterización y control de mezclas bituminosas. p. 21. Centro de Estudios y Experimentación de Obras Públicas (CEDEX), Madrid, 2000.

[11] Dirección General de Carreteras. Secciones de firme de la Instrucción de Carreteras IC. p. 36. Ministerio de Fomento. Ediciones Liteam, Madrid, 2002.

[12] J. Alarcón. Estudio del comportamiento de mezclas bituminosas recicladas en caliente en planta. Tesis Doctoral. p. 232. Universidad Politécnica de Cataluña. Escuela Técnica Superior de Ingenieros de Caminos, Canales y Puertos, Barcelona, 2003. 